\title{
AN EXISTENCE RESULT FOR A CLASS OF NONLINEAR FUNCTIONAL INTEGRAL EQUATIONS
}

\author{
KHALID LATRACH
}

Communicated by Jürgen Appell

\begin{abstract}
We consider a general nonlinear functional integral equation, and we prove the existence of solutions of this equation in the space of Lebesgue integrable functions on $\mathbb{R}^{+}$. Our analysis uses a recent version of Krasnosel'skii's fixed point theorem (Theorem 2.6) and the concept of the measure of weak noncompactness. In the appendix, we give an extension of Theorem 2.6 to expansive mappings.
\end{abstract}

1. Introduction. Nonlinear integral equations have a lot of applications in engineering, mechanics, economics, mathematical physics and so on (see, for example, $[\mathbf{4}, \mathbf{5}, \mathbf{1 0}, \mathbf{1 4}, \mathbf{2 8}]$ and the references therein). The typical equations which are often investigated are the Hammerstein integral equation and its generalization, the Urysohn integral equation (cf., $[3,4,5,7,10,14,15,16,22,23,26,28]$ ),

$$
\psi(t)=f(t, \psi(t))+\int_{I} \zeta(t, s, \psi(s)) d s, \quad t \in I,
$$

where $I$ is an interval in $\mathbb{R}$ (bounded or not) and $f: I \times \mathbb{R} \rightarrow \mathbb{R}$, $\zeta:, I \times I \times \mathbb{R} \rightarrow \mathbb{R}$ are given functions while $\psi(\cdot)$ is an unknown function. In the case where $I$ is bounded, the theory of (1.1) is well understood in the $L^{1}$ setting (see, for example, $[\mathbf{1 2}, \mathbf{2 1}, \mathbf{2 5}, \mathbf{2 7}, \mathbf{3 1}]$ ). However, the case of unbounded interval $I$ seems to be more difficult. Actually, in [6], an example of the measure of weak noncompactness on $L^{1}\left(\mathbb{R}^{+}\right)$ was constructed by Banaś and Knap. It furnished an adequate tool to settle such problems on unbounded intervals of $\mathbb{R}$. So, during the last decade, much work was done for a variety of nonlinear integral

2010 AMS Mathematics subject classification. Primary 47H10, 47H30.

Keywords and phrases. Functional integral equation, the Carathéodory conditions, integrable solutions, measure of weak noncompactness, fixed point theorem.

Received by the editors on April 30, 2014, and in revised form on January 19, 2015.

DOI:10.1216/JIE-2015-27-2-199

Copyright (ㅇ)2015 Rocky Mountain Mathematics Consortium 
equations on the space $L^{1}(I)$ where $I$ is an unbounded interval of $\mathbb{R}$ (see, for example, $[4,5,7,10,23,28]$ ).

Motivated by these works, we consider the following quite general nonlinear functional integral equation

$$
\psi(t)=f_{1}(t, \psi(t))+f_{2}\left(t, \int_{0}^{t} \zeta_{1}(t, s, \psi(s)) d s, \int_{0}^{+\infty} \zeta_{2}(t, s, \psi(s)) d s\right),
$$

where $f_{1}, f_{2}, \zeta_{1}$ and $\zeta_{2}$ are measurable functions while $\psi(\cdot)$ belongs to the space $L^{1}\left(\mathbb{R}^{+}\right)$. The goal of this paper is to discuss the solvability of equation (1.2) on the space $L^{1}\left(\mathbb{R}^{+}\right)$under general hypotheses on the functions $f_{1}, f_{2}, \zeta_{1}$ and $\zeta_{2}$. We will require that these functions satisfy the Carathéodory conditions and some other technical assumptions. Our analysis is based on a recent version of Krasnosel'skii's fixed point theorem established in [22] (cf., Theorem 2.6) and uses the concept of the measure of weak noncompactness on $L_{1}\left(\mathbb{R}^{+}\right)$. Our result seems to be new and covers many previous ones present in the literature, in particular, those obtained in $[4,5,7,10,23,26,28]$.

2. Preliminaries. This section is devoted to recalling some notions and results needed in the sequel. Let $\mathbb{R}$ be the set of real numbers, $\mathbb{R}^{+}$ the interval $[0,+\infty)$, and let

$$
\mathrm{X}:=L^{1}\left(\mathbb{R}^{+}, d x\right)
$$

be the space of Lebesgue integrable functions on the set $\mathbb{R}$ endowed with the standard norm

$$
\|\psi\|=\int_{0}^{+\infty}|\psi(t)| d t .
$$

We recall some facts concerning superposition operators required below. Let $I$ be an interval of $\mathbb{R}$. A function $f: I \times \mathbb{R} \rightarrow \mathbb{R}$ is said to satisfy the Carathéodory conditions on $I \times \mathbb{R}$ if

$$
\left\{\begin{array}{l}
t \longrightarrow f(t, x) \quad \text { is measurable on } I \text { for all } x \in \mathbb{R}, \\
x \longrightarrow f(t, x) \quad \text { is continuous on } \mathbb{R} \text { for almost all } t \in I .
\end{array}\right.
$$

If $f$ satisfies the Carathéodory conditions, then we can define the superposition (or Nemytskii) operator generated by $f, \mathcal{N}_{f}$, on the set of measurable real functions $\psi(\cdot)$ by $\left(\mathcal{N}_{f} \psi\right)(t)=f(t, \psi(t))$ for all $t \in I$. In 
$L^{p}$-spaces, the superposition operator has been extensively investigated (see, for example, [2, 21] and the bibliography therein). We recall the following result which states a basic fact for the theory of these operators in $L^{1}$-spaces.

Lemma 2.1. [2, 21]. Let $I$ be an interval of $\mathbb{R}$, and let $f: I \times \mathbb{R} \rightarrow \mathbb{R}$ be a function satisfying the Carathéodory conditions. The superposition operator $\mathcal{N}_{f}$ maps continuously the space $L^{1}(I)$ into itself if and only if

$$
|f(t, x)| \leq a(t)+b|x|, \quad \text { almost everywhere in } t, \text { for all } x \in \mathbb{R},
$$

where $b$ is a nonnegative constant and $a(\cdot)$ is a function of $L_{+}^{1}(I)$ where $L_{+}^{1}(I)$ stands for positive cone of the space $L^{1}(I)$.

This theorem is valid for both bounded and unbounded intervals. It was proved for bounded intervals by Krasnosel'skii [20]. A proof of this result for unbounded intervals appeared in [2].

Notation. Let $f$ be a function defined on a set $I$, and let $J$ be a nonempty subset of $I$. By $F_{\mid J}$, we denote the restriction of $f$ to $J$. For any subset $D$ of $\mathbb{R}$, by $\mu(D)$, we denote the Lebesgue measure of $D$.

We also need the following result due to Scorza-Dragoni concerning the structure of functions satisfying the Carathéodory conditions (see [9]).

Lemma 2.2. Let $I$ be a bounded interval, and let $f: I \times \mathbb{R} \rightarrow \mathbb{R}$ be a function satisfying the Carathéodory conditions. Then, for each $\varepsilon>0$, there exists a closed subset $D_{\varepsilon}$ of the interval $I$ such that $\mu\left(I \backslash D_{\varepsilon}\right) \leq \varepsilon$ and $f_{\mid D_{\varepsilon} \times \mathbb{R}}$ is continuous.

Let us now recall some facts about the Volterra linear operator on the space $X$. Denote by $\Pi$ the set

$$
\Pi=\{(t, s): 0 \leq s \leq t, s, t \in \mathbb{R}\}
$$


and let $\kappa$ be the function defined from $\Pi$ into $\mathbb{R}$ measurable with respect to both variables. Let $K$ be the operator defined for all $\psi \in \mathbf{X}$ by

$$
(K \psi)(t)=\int_{0}^{t} \kappa(t, s) \psi(s) d s, \quad t \geq 0 .
$$

The operator $K$ is called the Volterra integral operator. The following lemma gives a fundamental fact concerning this class of operators (see, for example, [31]).

Lemma 2.3. If the Volterra integral operator $K$ transforms the space $\mathrm{X}$ into itself, then it is continuous.

Let $Z$ be an infinite dimensional Banach space with the norm $\|\cdot\|$ and the zero element 0 . Let $\mathcal{B}(Z)$ denote the family of all nonempty bounded subsets of $Z$, and let $\mathcal{W}(Z)$ be the subset of $\mathcal{B}(\mathcal{Z})$ consisting of all weakly compact subsets of $Z$. For every real $r>0$, we denote by $B_{r}$ the closed ball of $Z$ centered at 0 with radius $r$.

Recall that the concept of a measure of weak noncompactness has been introduced by De Blasi [11] as follows:

$$
\left\{\begin{array}{l}
w: \mathcal{B}(Z) \longrightarrow \mathbb{R}^{+} \\
w(M):=\inf \left\{r>0: \text { there exists } Y \in \mathcal{W}(Z) \text { such that } M \subset Y+B_{r}\right\} .
\end{array}\right.
$$

In [1], Appell and De Pascale showed in $L_{1}(a, b)$ where $[a, b]$ is an interval of $\mathbb{R}$ that $w(\cdot)$ possesses the following simple form:

$$
w(M)=\limsup _{\varepsilon \rightarrow 0}\left\{\sup _{\psi \in M}\left[\int_{D}|\psi(t)| d t: \mu(D) \leq \varepsilon\right]\right\}
$$

for all bounded subsets $M$ of $L^{1}(a, b)$.

In [13], Dieudonné established the following characterization of weakly compact subsets of $L^{1}(\Omega)$ where $\Omega$ is an arbitrary subset of $\mathbb{R}^{n}, n \geq 1$. In the special case where $n=1$ and $\Omega=\mathbb{R}^{+}$, this result can be stated as follows:

Lemma 2.4. A bounded subset $M$ of $\mathrm{X}$ is relatively weakly compact if and only if 
(i) for any $\varepsilon>0$, there exists $\delta>0$ such that, if $\mu(D) \leq \delta$, then $\int_{D}|f(t)| d t<\varepsilon$ for all $f \in M$

(ii) for any $\varepsilon>0$, there exists $T>0$ such that $\int_{T}^{+\infty}|f(t)| d t \leq \varepsilon$ for all $f \in M$.

Using this lemma, Banaś and Knap [6] constructed a measure of weak noncompactness on the space $\mathbf{X}$ in the following way:

$$
\varnothing(M)=\varnothing_{1}(M)+\varnothing_{2}(M) \quad \text { for all } M \in \mathcal{B}(\mathrm{X}),
$$

where

$$
\emptyset_{1}(M)=\limsup _{\varepsilon \rightarrow 0}\left\{\sup _{\psi \in M}\left(\int_{D}|\psi(t)| d t: \text { meas }(D)<\varepsilon\right)\right\},
$$

and

$$
\emptyset_{2}(M)=\lim _{T \rightarrow+\infty}\left\{\sup _{\psi \in M}\left(\int_{T}^{+\infty}|\psi(t)| d t\right)\right\} .
$$

For completeness, we recall some useful properties of $\varnothing(\cdot)$ needed below (for the proofs we refer to [6]).

Lemma 2.5. Letting $M_{1}, M_{2} \in \mathcal{B}(\mathrm{X})$, then we have:

(i) $M_{1} \subseteq M_{2}$ implies $\varnothing\left(M_{1}\right) \leq \varnothing\left(M_{2}\right)$.

(ii) $\varnothing\left(\operatorname{co}\left(M_{1}\right)\right)=\varnothing\left(M_{1}\right)$.

(iii) $\varnothing\left(M_{1}+M_{2}\right) \leq \varnothing\left(M_{1}\right)+\varnothing\left(M_{2}\right)$.

(iv) If $\left(M_{n}\right)_{n \geq 1}$ is a sequence of nonempty, weakly closed subsets of $\mathrm{X}$ with $M_{1} \in \mathcal{B}(\mathrm{X})$ and $M_{1} \supseteq M_{2} \supseteq \cdots \supseteq M_{n} \supseteq \cdots$ with $\lim _{n \rightarrow \infty} w\left(M_{n}\right)=0$, then $\bigcap_{n=1}^{\infty} M_{n} \neq \emptyset$ and $\varnothing\left(\bigcap_{n=1}^{\infty} M_{n}\right)=0$.

Let $Z$ be a Banach space, and let $T$ be a nonlinear operator from $D(T) \subset Z$ into $Z$. The following condition is required in the sequel.

(A) If $\left(x_{n}\right)_{n \in \mathbb{N}} \subseteq D(T)$ is a weakly convergent sequence in $Z$, then $\left(T x_{n}\right)_{n \in \mathbb{N}}$ has a strongly convergent subsequence in $Z$.

The proof of our existence result will use the following fixed point theorem established in [22]. 
Theorem 2.6. Let $M$ be a nonempty bounded closed convex subset of a Banach space $Z$. Suppose that $A: M \rightarrow Z$ and $B: M \rightarrow Z$ such that:

(i) $A$ is continuous and satisfies (A),

(ii) there exists $\gamma \in[0,1[$ such that $w(A(S)+B(S)) \leq \gamma w(S)$ for all $S \subset M$,

(iii) $B$ is a strict contraction,

(iv) $A(M)+B(M) \subseteq M$.

Then there is $x \in M$ satisfying the equation $x=A(x)+B(x)$.

\section{Remark 2.7.}

(i) Evidently, this result remains valid if the condition $A(M)+$ $B(M) \subseteq M$ is replaced by the weaker one $(x=B x+A y, y \in$ $M) \Rightarrow x \in M$ (cf., [8]).

(ii) In Theorem 2.6, the operator $B$ is assumed to be a strict contraction. In fact, this result remains valid if $B$ belongs to other classes of operators. In the case where $B$ is a separate contractions [24] (it is a large class of functions which contains strictly the class of large contractions and strict contractions), this result was proved in [29]. An extension of this result to expansive mappings is given in the appendix.

3. Existence result. In this section, we are concerned with the solvability of the functional integral equation (1.2). To do so, we will impose suitable conditions on the functions involved in that equation. Namely, we assume

(i) $f_{1}: \mathbb{R}^{+} \times \mathbb{R} \rightarrow \mathbb{R}$ satisfies the Carathéodory conditions, and there are a function $0<\alpha(\cdot) \in \mathbf{X}$ and a constant $0 \leq \beta$ such that $\left|f_{1}(t, x)\right| \leq \alpha(t)+\beta|x|$ for $t \in \mathbb{R}^{+}$and for $x \in \mathbb{R}$;

(ii) $f_{1}$ is a strict contraction with respect to the second variable;

(iii) $f_{2}: \mathbb{R}^{+} \times \mathbb{R} \times \mathbb{R} \rightarrow \mathbb{R}$ satisfies the Carathéodory conditions, and there are a constant $\tau>0$ and a function $0 \leq \rho \in \mathbf{X}$ such that $\left|f_{2}(t, x, y)\right| \leq \rho(t)+\tau(|x|+|y|)$ for $t \in \mathbb{R}^{+}$and $x, y \in \mathbb{R}$;

(iv) $\zeta_{1}: \mathbb{R}^{+} \times \mathbb{R}^{+} \times \mathbb{R}, t \rightarrow \zeta_{1}(t, s, x)$ is measurable on $\mathbb{R}^{+}$for all $(s, x) \in \mathbb{R}^{+} \times \mathbb{R}$, and the function $(s, x) \rightarrow \zeta(t, s, x)$ is continuous on the set $\mathbb{R}^{+} \times \mathbb{R}$ for almost all $t \in \mathbb{R}^{+}$; 
(v) $\left|\zeta_{1}(t, s, x)\right| \leq \kappa_{1}(t, s)+\kappa_{2}(t, s)|x|$ for $(t, s, x) \in \mathbb{R}^{+} \times \mathbb{R}^{+} \times \mathbb{R}$ where $\kappa_{i}: \mathbb{R}^{+} \times \mathbb{R}^{+} \rightarrow \mathbb{R}^{+}, i=1,2$, satisfy the Carathéodory conditions; the linear operator $K$ defined by

$$
(K \psi)(t)=\int_{0}^{t} \kappa_{2}(t, s) \psi(s) d s
$$

transforms $\mathbf{X}$ into itself and $\int_{0}^{t} \kappa_{1}(t, s) d s \in \mathbf{X}$

(vi) $t \rightarrow \zeta_{2}(t, s, x)$ is measurable on $\mathbb{R}^{+}$for all $(s, x) \in \mathbb{R}^{+} \times \mathbb{R}$, and the function $(s, x) \rightarrow \zeta_{2}(t, s, x)$ is continuous on the set $\mathbb{R}^{+} \times \mathbb{R}$ for all $t \in \mathbb{R}^{+}$;

(vii) $\left|\zeta_{2}(t, s, x)\right| \leq \kappa_{3}(t, s)[\eta(s)+\nu|x|]$ for $(s, t, x) \in \mathbb{R}^{+} \times \mathbb{R}^{+} \times \mathbb{R}$, where $\nu$ is a nonnegative constant, $0 \leq \eta(\cdot) \in \mathbf{X}$ and $\kappa_{3}: \mathbb{R}^{+} \times \mathbb{R}^{+} \rightarrow \mathbb{R}^{+}$ is a measurable function such that the linear operator

$$
(\widehat{K} \psi)(t):=\int_{0}^{+\infty} \kappa_{3}(t, s) \psi(s) d s
$$

transforms $\mathrm{X}$ into itself and is bounded;

(viii) $\gamma:=\beta+\tau(\|K\|+\nu\|\widehat{K}\|)<1$.

Remark 3.1. It should be noticed that in condition (viii), $\|\kappa\|$ (respectively, $\|\widehat{K}\|)$ denotes the norm of the operator $K$ (respectively, $\widehat{K}$ ). The boundedness of $K$ (respectively, $\widehat{K}$ ) follows from Lemma 2.3 (respectively, hypothesis (vii)).

Now we are ready to state and prove our existence result.

Theorem 3.2. Assume that the hypotheses (i)-(viii) are satisfied. Then Problem (1.2) has at least one solution $\psi \in \mathrm{X}$.

Proof. Defining on $\mathrm{X}$ the operators

$$
\begin{aligned}
& (\Pi \psi)(t):=\int_{0}^{t} \zeta_{1}(t, s, \psi(s)) d s, \\
& (\Xi \psi)(t):=\int_{0}^{+\infty} \zeta_{2}(t, s, \psi(s)) d s,
\end{aligned}
$$

we see that the problem (1.2) may take the form

$$
\psi(t)=\left(\mathcal{N}_{f_{1}} \psi\right)(t)+(\mathcal{H} \psi)(t)
$$


where $\mathcal{N}_{f_{1}}$ is the superposition operator generated by the function $f_{1}$ and $\mathcal{H}$ is defined by

$$
(\mathcal{H} \psi)(t)=f_{2}(t,(\Pi \psi)(t),(\Xi \psi)(t)) .
$$

The proof uses Theorem 2.6 and will be derived in several steps.

Step 1. Continuity of the operators $\mathcal{N}_{f_{1}}$ and $\mathcal{H}$. The continuity of $\mathcal{N}_{f_{1}}$ follows from hypothesis (i) and Lemma 2.1 .

Now we shall prove the continuity of the operators $\Pi$ and $\Xi$. To do so, let $\left(\psi_{n}\right)_{n}$ be a sequence in $\mathbf{X}$ which converges to $\varphi \in \mathbf{X}$. According to [18, Theorem 6.11], there exist a function $\varphi(\cdot) \in \mathrm{X}$ and a subsequence $\left(\psi_{n_{k}}\right)_{k}$ which converges to $\psi$ almost everywhere on $\mathbb{R}^{+}$such that

$$
\left|\psi_{n_{k}}(s)\right| \leq \varphi(s) \quad \text { almost everywhere in } \mathbb{R}^{+} .
$$

Since $\psi_{n_{k}} \rightarrow \psi$ almost everywhere on $\mathbb{R}^{+}$, the use of the continuity of $\zeta_{1}$ with respect to the third variable gives:

$$
\zeta_{1}\left(t, s, \psi_{n_{k}}(s)\right) \longrightarrow \zeta_{1}(t, s, \psi(s)) \text { for almost all } s, t \in \mathbb{R}^{+} .
$$

Next, the use of the estimate (3.1) together with assumption (v) yields

$$
\left|\zeta_{1}\left(t, s, \psi_{n_{k}}(s)\right)\right| \leq \kappa_{1}(t, s)+\kappa_{2}(t, s) \psi_{n_{k}}(s) .
$$

Accordingly, applying the Lebesgue dominated convergence theorem we get

$$
\left(\Pi \psi_{n_{k}}\right)(t) \longrightarrow(\Pi \psi)(t) \quad \text { for almost all } t \in \mathbb{R}^{+} .
$$

By hypothesis (v), it is clear that

$$
\left|\left(\Pi \psi_{n_{k}}\right)(t)\right| \leq \int_{0}^{t} \kappa_{1}(t, s) d s+\int_{0}^{t} \kappa_{2}(t, s) \varphi(s) d s
$$

for almost all $t \in \mathbb{R}^{+}$. Since, again by (v),

$$
\int_{0}^{+\infty} \int_{0}^{t} \kappa_{1}(t, s) d s d t+\int_{0}^{+\infty} \int_{0}^{t} \kappa_{2}(t, s) \varphi(s) d s d t<+\infty,
$$

the use of Lebesgue's dominated convergence theorem gives

$$
\left\|\Pi \psi_{n_{k}}-\Pi \psi\right\|_{\mathrm{x}} \longrightarrow 0 .
$$

Since any sequence $\left(\psi_{n}\right)_{n}$ of $\mathbf{X}$ converging to $\psi$ in $\mathbf{X}$ has a converging subsequence $\left(\psi_{n_{k}}\right)_{k}$ such that $\Pi \psi_{n_{k}} \rightarrow \Pi \psi$ in $\mathbf{X}$, we infer that $\Pi$ is continuous. 
Similar reasoning proves that $\Xi$ is a continuous operator from $\mathrm{X}$ into itself.

The continuity of $\mathcal{H}$ follows from the continuity of $\Pi, \Xi$, hypotheses (iii)-(vii) and Lemma 2.1.

Step 2. There exists a real number $r>0$ such that $\mathcal{N}_{f_{1}}\left(B_{r}\right)+$ $\mathcal{H}\left(B_{r}\right) \subseteq B_{r}$. Indeed, if $\psi, \varphi \in \mathrm{X}$, then we have

$$
\begin{aligned}
\left\|\mathcal{N}_{f_{1}} \psi+\mathcal{H} \varphi\right\| \leq & \int_{0}^{+\infty}\left|f_{1}(t, \psi(t))\right| d t \\
& +\int_{0}^{+\infty}\left|f_{2}(t,(\Pi \varphi)(t),(\Xi \varphi)(t))\right| d t \\
= & \int_{0}^{+\infty}\left|f_{1}(t, \psi(t))\right| d t \\
& +\int_{0}^{+\infty} \mid f_{2}\left(t, \int_{0}^{t} \zeta_{1}(t, s, \varphi(s)) d s,\right. \\
\leq & \|\alpha\|+\beta\|\psi\|+\|\rho\|+\tau \int_{0}^{+\infty}|(\Pi \psi)(t)| d t \\
& +\tau \int_{0}^{+\infty}|(\Xi \psi)(t)| d t .
\end{aligned}
$$

If $\left\|\kappa_{1}\right\|=\int_{0}^{+\infty} \int_{0}^{t} \kappa_{1}(t, s) d s d t$, then easy calculations give

$$
\int_{0}^{+\infty}|(\Pi \psi)(t)| d t \leq\left\|\kappa_{1}\right\|+\|K\|\|\varphi\|,
$$

and

$$
\begin{aligned}
\int_{0}^{+\infty}|(\Xi \psi)(t)| d t \leq & \int_{0}^{+\infty} \int_{0}^{+\infty} \kappa_{3}(t, s) \eta(s) d s d t \\
& +\nu \int_{0}^{+\infty} \int_{0}^{+\infty} \kappa_{3}(t, s)|\varphi(s)| d s d t \\
\leq & \|\widehat{K}\|(\|\eta\|+\nu\|\varphi\|)
\end{aligned}
$$

Consequently,

$$
\left\|\mathcal{N}_{f_{1}} \psi+\mathcal{H} \varphi\right\| \leq\|\alpha\|+\beta\|\psi\|+\|\rho\|
$$




$$
\begin{aligned}
& +\tau\left(\left\|\kappa_{1}\right\|+\|K\|\|\varphi\|\right) \\
& +\tau\|\widehat{K}\|(\|\eta\|+\nu\|\varphi\|) \\
\leq & \|\alpha\|+\|\rho\|+\tau\left(\left\|\kappa_{1}\right\|+\|\widehat{K}\|\|\eta\|\right) \\
& +(\beta+\tau\|K\|+\tau \nu\|\widehat{K}\|) .
\end{aligned}
$$

Let $r$ be the real defined by

$$
r:=\frac{\|\alpha\|+\|\rho\|+\tau\left(\left\|\kappa_{1}\right\|+\|\widehat{K}\|\|\eta\|\right)}{1-(\beta+\tau\|K\|+\tau \nu\|\widehat{K}\|)} .
$$

It is clear that $r>0$ (use condition (viii)). Now the estimate (3.2) implies that $\mathcal{N}_{f_{1}}\left(B_{r}\right)+\mathcal{H}\left(B_{r}\right) \subset B_{r}$.

Step 3. There exists $\gamma \in[0,1)$ such that $\varnothing\left(\mathcal{N}_{f_{1}}(S)+\mathcal{H}(S)\right) \leq \gamma \varnothing(S)$ for all bounded subset $S$ of $\mathrm{X}$.

Let $\varepsilon>0$ be an arbitrary real, and take a nonempty set $D \subset \mathbb{R}^{+}$ such that $\mu(D) \leq \varepsilon$. Let $S$ be a bounded subset of X. For $\varphi, \psi \in S$, we have

$$
\begin{aligned}
\int_{D}\left|\left(\mathcal{N}_{f_{1}} \varphi\right)(t)+(\mathcal{H} \psi)(t)\right| d t \leq & \int_{D}\left|f_{1}(t, \varphi(t))\right| d t \\
& +\int_{D} \mid f_{2}\left(t, \int_{0}^{t} \zeta_{1}(t, s, \varphi(s)) d s\right. \\
& \left.\int_{0}^{+\infty} \zeta_{2}(t, s, \varphi(s)) d s\right) d t \\
\leq & \int_{D} \alpha(t) d t+\beta \int_{D}|\varphi(t)| d t+\int_{D} \rho(t) d t \\
& +\tau \int_{D}\left|\int_{0}^{t} \zeta_{1}(t, s, \psi(s)) d s\right| d t \\
& +\tau \int_{D}\left|\int_{0}^{+\infty} \zeta_{2}(t, s, \psi(s)) d s\right| d t \\
\leq & \int_{D} \alpha(t) d t+\beta \int_{D}|\varphi(t)| d t+\int_{D} \rho(t) d t \\
& +\tau \int_{D} \int_{0}^{t} \kappa_{1}(t, s) d s d t \\
& +\tau \int_{D} \int_{0}^{+\infty} \kappa_{2}(t, s)|\varphi(s)| d s d t
\end{aligned}
$$




$$
\begin{aligned}
& +\tau \int_{D} \int_{0}^{+\infty} \kappa_{3}(t, s) \eta(s) d s d t \\
& +\int_{D} \int_{0}^{+\infty} \kappa_{3}(t, s)|\psi(s)| d s d t \\
& \leq \int_{D} \alpha(t) d t+\beta \int_{D}|\varphi(t)| d t+\int_{D} \rho(t) d t \\
& +\tau \int_{D} \int_{0}^{t} \kappa_{1}((t, s) d s d t \\
& +\tau\|\widehat{K}\| \int_{D}|\eta(t)| d t \\
& +\tau(\|K\|+\nu\|\widehat{K}\|) \int_{D}|\psi(t)| d t .
\end{aligned}
$$

Next, using the fact that sets consisting in one element are weakly compact we infer that

$$
\begin{aligned}
\emptyset_{1}\left(\mathcal{N}_{f_{1}}(S)+\mathcal{H}(S)\right) & \leq(\beta+\tau(\|K\|+\nu\|\widehat{K}\|)) \emptyset_{1}(S) \\
& =\gamma \emptyset_{1}(S) .
\end{aligned}
$$

On the other hand, for $T \in \mathbb{R}^{+}$, we have $\int_{T}^{\infty}\left|\left(\mathcal{N}_{f_{1}} \varphi\right)(t)+(\mathcal{H} \psi)(t)\right| d t$ is sufficiently small for $T$ sufficiently large. So, using hypotheses (iii), (v) and (vii) for $\varphi, \psi \in S$, we have:

$$
\begin{aligned}
\int_{T}^{\infty}\left|\left(\mathcal{N}_{f_{1}} \varphi\right)(t)+(\mathcal{H} \psi)(t)\right| d t \leq & \int_{T}^{+\infty} \alpha(t) d t \\
& +\beta \int_{T}^{+\infty}|\varphi(t)| d t+\int_{T}^{+\infty} \rho(t) d t \\
& +\tau \int_{T}^{+\infty} \int_{0}^{t} \kappa_{1}(t, s) d s d t \\
& +\int_{T}^{+\infty} \int_{0}^{t} \kappa_{2}(t, s)|\psi(s)| d s d t \\
& +\tau \int_{T}^{+\infty} \int_{0}^{+\infty} \kappa_{3}(t, s) \eta(s) d s d t \\
& +\nu \int_{T}^{+\infty} \int_{0}^{+\infty} \kappa_{3}(t, s)|\psi(s)| d s d t \\
\leq & \int_{T}^{+\infty} \alpha(t) d t+\beta \int_{T}^{+\infty}|\varphi(t)| d t
\end{aligned}
$$




$$
\begin{aligned}
& +\int_{T}^{+\infty} \rho(t) d t+\tau \int_{T}^{+\infty} \int_{0}^{t} \kappa_{1}(t, s) d s d t \\
& +\tau\|\widehat{K}\| \int_{T}^{+\infty} \eta(t) d t+\tau(\|K\|+\nu\|\widehat{K}\|) \\
& \quad \times \int_{T}^{+\infty}|\psi(t)| d t .
\end{aligned}
$$

According to the definition of $\phi_{2}$, we infer that

$$
\varnothing_{2}(\mathcal{H}(S)) \leq(\beta+\tau(\|K\|+\nu\|\widehat{K}\|)) \emptyset_{2}(S)=\gamma \varnothing_{2}(S) .
$$

Therefore, (3.4) and (3.5) imply

$$
\varnothing\left(\mathcal{N}_{f_{1}}(S)+\mathcal{H}(S)\right) \leq \gamma \varnothing(S) \text { for all } S \in \mathcal{B}(\mathrm{X})
$$

Step 4. There exists a nonempty convex weakly compact subset $M_{\infty}$ of $B_{r}$ such that $\mathcal{N}_{f_{1}}\left(M_{\infty}\right)+\mathcal{H}\left(M_{\infty}\right) \subseteq M_{\infty}$. Indeed, set

$$
M^{0}=B_{r} \quad \text { and } \quad M^{n+1}=\overline{\mathrm{co}}\left(\mathcal{N}_{f_{1}}\left(M^{n}\right)+\mathcal{H}\left(M^{n}\right)\right) \quad n=1,2, \ldots,
$$

where $\overline{\mathrm{co}}(A)$ denotes the closed convex hull of the set $A$. It is clear that family $\left(M^{n}\right)_{n}$ consists of nonempty closed convex subset of $\mathrm{X}$ satisfying $M^{n+1} \subset M^{n}, n=1,2, \ldots$ So, for all $n \in \mathbb{N} \backslash\{0\}$, we have

$$
\varnothing\left(\mathcal{N}_{f_{1}}\left(M^{n}\right)+\mathcal{H}\left(M^{n}\right)\right) \leq \gamma \varnothing\left(M^{n}\right) \leq \cdots \leq \gamma^{n} \varnothing\left(M^{0}\right) .
$$

Using the fact that $\gamma<1$, we get $\lim _{n \rightarrow+\infty} \varnothing\left(M^{n}\right)=0$. Set

$$
M_{\infty}=\bigcap_{0}^{+\infty} M^{n} .
$$

Applying Lemma 2.5 (iv), we conclude that $M_{\infty}$ is a nonempty convex weakly compact subset of $\mathrm{X}$; moreover,

$$
\mathcal{N}_{f_{1}}\left(M_{\infty}\right)+\mathcal{H}\left(M_{\infty}\right) \subseteq M_{\infty} .
$$

Step 5. H satisfies the condition (A) on the set $M_{\infty}$. The proof of this step is given in the appendix.

Step 6. Existence of a solution to problem (1.2). Indeed, let $r$ be the real defined by (3.3) and restrict the problem (1.2) to the set $M_{\infty} \subset B_{r}$. It is clear that $\mathcal{N}_{f_{1}}$ is a strict contraction on $M_{\infty}$ (use 
hypothesis (ii)). Now, using the results of Steps 1, 2, 3 and 5, one sees that the operators $\mathcal{N}_{f_{1}}$ and $\mathcal{H}$ satisfy the hypotheses of Theorem 2.6. Accordingly, problem (1.2) has at least a solution in $M_{\infty} \subset B_{r}$.

\section{Remark 3.3.}

(i) Note that, as in many recent works (see, for example, [19, 10, 23]), instead of investigating equation (1.2), we can consider the equation

$$
\begin{aligned}
\psi(t)=f_{1}\left(t, \psi\left(\phi_{1}(t)\right)\right)+f_{2}\left(t, \int_{0}^{\phi_{2}(t)} \zeta_{1}\left(t, s, \psi\left(\phi_{3}(s)\right)\right) d s\right. & \\
& \left.\int_{0}^{+\infty} \zeta_{2}\left(t, s, \psi\left(\phi_{4}(s)\right)\right) d s\right)
\end{aligned}
$$

where $\phi_{i}, i=1,2,3,4$, are functions from $\mathbb{R}^{+}$into $\mathbb{R}^{+}$. Our existence result remains valid. It suffices to suppose that $\phi_{2}$ is increasing, $\phi_{i}$ are absolutely continuous functions, $\lim _{t \rightarrow+\infty} \phi_{i}(t)=+\infty$, and there exist positive constants $\alpha_{i}>0$ such that $\phi_{i}^{\prime}(t) \geq \alpha_{i}$ for all $t \in \mathbb{R}^{+}, i=1,2,3,4$.

(ii) Our existence result holds true if we suppose that the function $f_{1}$ is a large contraction, a separate contraction or expansive and onto with respect to the second variable (see Theorem 4.2).

(iii) Finally, we note that Theorem 3.2 remains valid if the operator $\Pi$ is defined by

$$
(\Pi \psi)(t)=\int_{0}^{+\infty} \zeta_{1}(t, s, \psi(s)) d s .
$$

4. Appendix. The proof of Step 5 uses Scorza-Dragoni's theorem (Lemma 2.2), and it is an adaptation to our case of the arguments used in $[4,5]$ (see also $[\mathbf{2 3}, \mathbf{2 8}]$ ). It seems that these arguments were first used by Emmanuele [15, 17].

Proof of Step 5. $\mathcal{H}$ satisfies condition $(\mathrm{A})$ on the set $M_{\infty}$. Indeed, let $\varepsilon>0$, and consider an arbitrary sequence $\left(\psi_{n}\right)_{n}$ of $M_{\infty}$. Since $M_{\infty}$ is weakly compact, by Lemma 2.4 , there exists $\tau>0$ such that

$$
\int_{\tau}^{+\infty}\left|\psi_{n}(t)\right| d t \leq \frac{\varepsilon}{6} .
$$


Since $\mathcal{H}\left(M_{\infty}\right) \subset M_{\infty}$, we infer that

$$
\int_{\tau}^{+\infty}\left|\mathcal{H}\left(\psi_{n}(t)\right)\right| d t \leq \frac{\varepsilon}{6} .
$$

Consider the functions

$$
\begin{array}{ll}
\left.f_{2}\right|_{[0, \tau] \times \mathbb{R}^{+} \times \mathbb{R}}, & \left.\zeta_{1}\right|_{[0, \tau] \times \mathbb{R}^{+} \times \mathbb{R}}, \\
\left.\zeta_{2}\right|_{[0, \tau] \times \mathbb{R}^{+} \times \mathbb{R}}, & \eta_{[0, \tau]},
\end{array}
$$

and

$$
\kappa_{i[0, \tau] \times \mathbb{R}^{+}}, \quad i=1,2,3 .
$$

According to Lusin's theorem and Lemma 2.2, there exists a subset $D_{\varepsilon}$ of $\mathbb{R}^{+}$such that $\mu\left([0, \tau] \backslash D_{\varepsilon}\right) \leq \varepsilon$ and

$$
\begin{array}{ll}
\left.f_{2}\right|_{D_{\varepsilon} \times \mathbb{R}^{+} \times \mathbb{R}^{\prime}}, & \left.\zeta_{1}\right|_{D_{\varepsilon} \times \mathbb{R}^{+} \times \mathbb{R}^{\prime}}, \\
\left.\zeta_{2}\right|_{D_{\varepsilon} \times \mathbb{R}^{+} \times \mathbb{R}^{\prime}}, & \zeta_{D_{\varepsilon} \times \mathbb{R}^{+}}
\end{array}
$$

and

$$
\left.\kappa_{i}\right|_{D_{\varepsilon} \times \mathbb{R}^{+}}, \quad i=1,2,3,
$$

are continuous. Set

$$
\Lambda_{i}:=\sup _{(t, s) \in D_{\varepsilon} \times[0, \tau]} \kappa_{i}(t, s), \quad i=1,2,3 .
$$

For $\psi \in B_{r}$ and $t \in D_{\varepsilon}$, we have

$$
\begin{aligned}
\int_{0}^{t}\left|\zeta_{1}(t, s, \psi(t))\right| d s \leq & \int_{0}^{t} \kappa_{1}(t, s) d s \\
& +\int_{0}^{t} \kappa_{2}(t, s)|\psi(s)| d s \\
\leq & \tau \sup _{(t, s) \in D_{\varepsilon} \times[0, \tau]} \kappa_{1}(t, s) \\
& +\sup _{(t, s) \in D_{\varepsilon} \times[0, \tau]} \kappa_{2}(t, s) \times \int_{0}^{t}|\psi(s)| d s \\
\leq & \tau \Lambda_{1}+r \Lambda_{2}
\end{aligned}
$$


and

$$
\begin{aligned}
\int_{0}^{+\infty}\left|\zeta_{2}(t, s, \psi(t))\right| d s \leq & \int_{0}^{+\infty} \kappa_{3}(t, s) \eta(s) d s \\
& +\int_{0}^{+\infty} \kappa_{3}(t, s) \nu|\psi(s)| d \\
\leq & \Lambda_{3}\left[\sup _{s \in D_{\varepsilon}} \eta(s) \tau+\nu r\right]
\end{aligned}
$$

Set

$$
M_{\varepsilon}:=\tau \Lambda_{1}+r \Lambda_{2} \quad \text { and } \quad \widehat{M}_{\varepsilon}:=\Lambda_{3}\left[\sup _{s \in D_{\varepsilon}} \eta(s) \tau+\nu r\right]
$$

Let us take (arbitrary reals) $t_{1}, t_{2} \in D_{\varepsilon}$ and suppose that $t_{1}<t_{2}$. Using the uniform continuity of $\left.\zeta_{1}\right|_{D_{\varepsilon} \times[0, \tau] \times\left[-M_{\varepsilon}, M_{\varepsilon}\right]}$, we can write:

$$
\begin{aligned}
\mid & \int_{0}^{t_{2}} \zeta_{1}\left(t_{2}, s, \psi_{n}(s)\right) d s-\int_{0}^{t_{1}} \zeta_{1}\left(t_{2}, s, \psi_{n}(s)\right) d s \\
\leq & \left|\int_{0}^{t_{2}} \zeta_{1}\left(t_{2}, s, \psi_{n}(s)\right) d s-\int_{0}^{t_{2}} \zeta_{1}\left(t_{1}, s, \psi_{n}(s)\right) d s\right| \\
& +\left|\int_{t_{1}}^{t_{2}} \zeta_{1}\left(t_{2}, s, \psi_{n}(s)\right) d s\right| \\
\leq & \left|\int_{0}^{\tau} \zeta_{1}\left(t_{2}, s, \psi_{n}(s)\right) d s-\int_{0}^{\tau} \zeta_{1}\left(t_{1}, s, \psi_{n}(s)\right) d s\right| \\
& +\left|\int_{t_{1}}^{t_{2}} \zeta_{1}\left(t_{2}, s, \psi_{n}(s)\right) d s\right| \\
\leq & \int_{[0, \tau] \backslash D_{\varepsilon}}\left|\zeta_{1}\left(t_{2}, s, \psi_{n}(s)\right)\right| d s \\
& +\int_{[0, \tau] \backslash D_{\varepsilon}}\left|\zeta_{1}\left(t_{1}, s, \psi_{n}(s)\right)\right| d s \\
& +\left|\int_{D_{\varepsilon}}^{t_{2}} \zeta_{1}\left(t_{2}, s, \psi_{n}(s)\right) d s-\int_{D_{\varepsilon}} \zeta_{1}\left(t_{1}, s, \psi_{n}(s)\right) d s\right| \\
& +\int_{t_{1}}^{\kappa_{1}} \kappa_{1}\left(t_{2}, s\right) d s+\iint_{t_{1}}^{t_{2}} \kappa_{2}\left(t_{2}, s\right)\left|\psi_{n}(s)\right| d s \\
\leq & \varnothing^{\zeta_{1}}\left(\left|t_{2}-t_{1}\right|\right)+2 \Lambda_{1}\left|[0, \tau] \backslash D_{\varepsilon}\right|
\end{aligned}
$$




$$
\begin{aligned}
& +2 \Lambda_{2} \int_{[0, \tau] \backslash D_{\varepsilon}}\left|\psi_{n}(s)\right| d s+\Lambda_{1}\left|t_{2}-t_{1}\right| \\
& +\Lambda_{2} \int_{t_{1}}^{t_{2}}\left|\psi_{n}(s)\right| d s \\
\leq & \phi^{\zeta_{1}}\left(\left|t_{2}-t_{1}\right|\right)+2 \Lambda_{1}\left|[0, \tau] \backslash D_{\varepsilon}\right|+2 \Lambda_{2} r\left|[0, \tau] \backslash D_{\varepsilon}\right| \\
& +\Lambda_{1}\left|t_{2}-t_{1}\right|+\Lambda_{2} \int_{t_{1}}^{t_{2}}\left|\psi_{n}(s)\right| d s
\end{aligned}
$$

where $\varnothing^{\zeta_{1}}\left(\left|t_{2}-t_{1}\right|\right)$ denotes the modulus of continuity of $\zeta_{1}$ on the set $D_{\varepsilon} \times[0, \tau] \times\left[-M_{\varepsilon}, M_{\varepsilon}\right]$. Observe that the weak compactness of the set $\left\{\psi_{n}: n \in \mathbb{N}\right\}$ together with Lemma 2.4 implies that the numbers $\int_{t_{1}}^{t_{2}}\left|\psi_{n}(s)\right| d s$ are arbitrary small provided the number $t_{2}-t_{1}$ is small enough. This proves that the right hand side of the previous inequality tends to zero independent of $n$ as $t_{2}-t_{1}$ tends to zero. Therefore, using (4.2), we conclude that the sequence

$$
\left(\int_{0}^{t}\left|\zeta_{1}\left(t, s, \psi_{n}(s)\right)\right| d s\right)_{n \in n \mathbb{N}}
$$

is equibounded and equicontinuous in the space $C\left(D_{\varepsilon}\right)$ (the space of all continuous function on $D_{\varepsilon}$ ). Similar reasoning using (4.3) and the same arguments as above proves that the sequence

$$
\left(\int_{0}^{+\infty}\left|\zeta_{2}\left(t, s, \psi_{n}(s)\right)\right| d s\right)_{n \in \mathbb{N}}
$$

is equibounded and equicontinuous in the space $C\left(D_{\varepsilon}\right)$. Next, using the fact that $\left.f_{2}\right|_{D_{\varepsilon} \times\left[-M_{\varepsilon}, M_{\varepsilon}\right] \times\left[-\widehat{M}_{\varepsilon}, \widehat{M}_{\varepsilon}\right]}$ is uniformly continuous, we infer that the sequence $\left(\mathcal{H}\left(\psi_{n}\right)\right)_{n \in \mathbb{N}}$ is equibounded on $D_{\varepsilon}$. Applying the Arzèla-Ascoli theorem we see that the set $\left\{\mathcal{H}\left(\psi_{n}\right), n=1,2, \ldots\right\}$ forms a relatively compact set in the space $C\left(D_{\varepsilon}\right)$.

It should be noticed that the above results do not depend on the choice of $\varepsilon$. Therefore, we can construct a sequence $\left(D_{1 / k}\right)_{k \in \mathbb{N}}$ of closed subsets of interval $[0, \tau]$ such that

$$
\mu\left([0, \tau] \backslash D_{1 / k}\right) \longrightarrow 0 \quad \text { as } k \rightarrow+\infty,
$$

and the set $\left\{\mathcal{H}\left(\psi_{n}\right), n=1,2, \ldots\right\}$ is relatively compact in every space $C\left(D_{1 / k}\right)$. Passing to a subsequence, if necessary, we can assume that 
$\left(\mathcal{H}\left(\psi_{n}\right)\right)_{n}$ is a Cauchy sequence in each space $C\left(D_{1 / k}\right), k=1,2, \ldots$

Next, using the fact that $\mathcal{H}\left(M_{\infty}\right)$ is relatively weakly compact (because $M_{\infty}$ is weakly compact and $\left.\mathcal{H}\left(M_{\infty}\right) \subset M_{\infty}\right)$, we can choose $\delta>0$ such that, for each closed subset $D$ of the interval $[0, \tau]$ satisfying $\mu([0, \tau] \backslash D) \leq \delta$, we have

$$
\int_{[0, \tau] \backslash D}|\mathcal{H}(\psi(s))| d s \leq \frac{\varepsilon}{6}
$$

for any $x \in M_{\infty}$. Keeping in mind the fact that $\left(\mathcal{H}\left(\psi_{n}\right)\right)_{n \in \mathbb{N}}$ is a Cauchy sequence in each space $C\left(D_{1 / k}\right)$, we can choose an integer $k_{0}$ such that $\mu\left([0, \tau] \backslash D_{1 / k_{0}}\right) \leq \delta$ and, for arbitrary integers $n, m \geq k_{0}$, the following inequality holds:

$$
\left|\left(\mathcal{H} \psi_{n}\right)(t)-\left(\mathcal{H} \psi_{m}\right)(t)\right| \leq \frac{\varepsilon}{3 \mu\left(D_{1 / k_{0}}\right)}
$$

for any $t \in D_{1 / k_{0}}$. Combining (4.1), (4.5) and (4.6), we get

$$
\begin{aligned}
\left\|\mathcal{H}\left(\psi_{n}\right)-\mathcal{H}\left(\psi_{m}\right)\right\|= & \int_{0}^{+\infty}\left|\mathcal{H}\left(x_{n}\right)(t)-\mathcal{H}\left(x_{m}\right)(t)\right| d t \\
= & \int_{\tau}^{+\infty}\left|\mathcal{H}\left(\psi_{n}\right)(t)-\mathcal{H}\left(\psi_{m}\right)(t)\right| d t \\
& +\int_{D_{1 / k_{0}}}\left|\mathcal{H}\left(\psi_{n}\right)(t)-\mathcal{H}\left(\psi_{m}\right)(t)\right| d t \\
& +\int_{[0, \tau] \backslash D_{1 / k_{0}}}\left|\mathcal{H}\left(\psi_{n}\right)(t)-\mathcal{H}\left(x_{m}\right)(t)\right| d t \\
\leq & \varepsilon .
\end{aligned}
$$

This proves that $\left(\mathcal{H}\left(\psi_{n}\right)\right)_{n \in \mathbb{N}}$ is a Cauchy sequence in $\mathbf{X}$. The completeness of $\mathrm{X}$ ensures that $\mathcal{H}$ satisfies the condition $(\mathrm{A})$.

In the remainder of this appendix we extend Theorem 2.6 to the class of expansive mappings.

Let $(Z, d)$ be a metric space, and let $M$ be a subset of $Z$. A mapping $B: M \rightarrow Z$ is said to be expansive, if there exists a constant $\mu>1$ such that

$$
d(B x, B y) \geq \varrho d(x, y), \quad \text { for all } x, y \in M .
$$


The following result gives an important property of expansive mappings.

Lemma 4.1. [30, Theorem 2.1]. Let $M$ be a nonempty closed subset of a Banach space $Z$, and let $B$ be a function from $M$ into $Z$. If $B$ is expansive and onto, then $B$ possesses a unique fixed point belonging to $M$.

The following statement is an improvement of Theorem 2.6.

Theorem 4.2. Let $M$ be a nonempty bounded closed convex subset of a Banach space $Z$. Suppose that $A: M \rightarrow Z$ and $B: M \rightarrow Z$ satisfy conditions (a), (b) and (d) of Theorem 2.6. Further, if $B$ is expansive and onto, then there is $x \in M$ such $x=A(x)+B(x)$.

Proof. For any $z \in M$, the map $\pi$ from $M$ into $Z$ defined by $\pi(x)=$ $B(x)+z$ satisfies $\left\|\pi(x)-\pi\left(x^{\prime}\right)\right\|=\left\|B(x)-B\left(x^{\prime}\right)\right\| \geq \varrho\left\|x-x^{\prime}\right\|$. So, it is expansive and onto. Thus, Lemma 4.1 shows that the equation $x=B(x)+z$ has a unique solution $u(z)$ in $M$. Therefore, $u$ defines a bijective function from $M$ into itself. Next, let $y_{1}, y_{2} \in M$. Using

$$
B\left(u\left(y_{1}\right)\right)+y_{1}=u\left(y_{1}\right) \text { and } B\left(u\left(y_{2}\right)\right)+y_{2}=u\left(y_{2}\right),
$$

we arrive at the inequality

$$
\mid B\left(u\left(y_{1}\right)\right)-B\left(u\left(y_{2}\right)\right)\|\leq\| y_{1}-y_{2}\|+\| u\left(y_{1}\right)-u\left(y_{2}\right) \| .
$$

Since $B$ is expansive, there exists a constant $\varrho>1$ such that

$$
\left\|B\left(u\left(y_{1}\right)\right)-B\left(u\left(y_{2}\right)\right)\right\| \geq \varrho\left\|u\left(y_{1}\right)-u\left(y_{2}\right)\right\|,
$$

and, consequently,

$$
\left\|u\left(y_{1}\right)-u\left(y_{2}\right)\right\| \leq \frac{1}{\varrho-1}\left\|y_{1}-y_{2}\right\| .
$$

This implies that the map $u: M \rightarrow M$ is continuous. Using the continuity of $A$ we deduce that $u \circ A$ is continuous. The remainder of the proof is now similar to that of [22, Theorem 2.1]. 


\section{REFERENCES}

1. J. Appell and E. De Pascale, Su alcuni parametri connessi con la misura di non compattezza di Hausdorff in spazi di funzioni misurabili, Boll. Mat. Ital. 3 (1984), 497-515.

2. J. Appell and P.P. Zabrejko, Nonlinear superposition operators, Cambridge University Press, Cambridge, 1990.

3. J. Banaś, Integrable solutions of Hammerstein and Uryshon integral equations, J. Aust. Math. Soc. 46 (1989), 61-68.

4. J. Banaś and A. Chlebowicz, On existence of integrable solutions of a functional integral equation under Carathéodory condition, Nonlin. Anal. 70 (2009), $3172-3179$.

5. - On integrable solutions of nonlinear Volterra integral equations under Carathéodory conditions, Bull. Lond. Math. Soc. 41 (2009), 1073-1084.

6. J. Banaś and Z. Knap, Measure of weak noncompactness and nonlinear integral equations of convolution type, J. Math. Anal. Appl. 146 (1990), 353-362.

7. J. Banaś and M. Paslawska-Poludniak, Monotonoc solutions of Urysohn integral equations on unbounded interval, Comp. Math. Appl. 47 (2004), 19471954.

8. T.A. Burton, A fixed point theorem of Krasnosel'skii, Appl. Math. Lett. 11 (1998), 85-88.

9. Castaing, Une nouvelle extension du théorème de Dragoni-Scorza, C.R. Acad. Sci. Paris 271 (1970), 396-398.

10. M.A. Darwish, On a perturbed functional integral equation of Uryshon type, Appl. Math. Comp. 218 (2012), 8800-8805.

11. F.S. De Blasi, On a property of the unit sphere in Banach spaces, Bull. Math. Soc. Sci. Math. Roum. 21 (1977), 259-262.

12. K. Deimling, Nonlinear functinal analysis, Springer Verlag, New York, 1985.

13. J. Dieudonné, Sur les espaces de Khöthe, J. Anal. Math. 1 (1951), 81-115.

14. W.G. El-Sayed, A.A. El Bary and M.A. Darwish, Solvability of Uryshon integral equation, Appl. Math. Comp. 145 (2003), 487-493.

15. G. Emmanuele, Integrable solutions of a functional-integral equation, J. Int. Equat. Appl. 4 (1992), 89-94.

16. An existence theorem for Hammerstein integral equations, Portug. Math. 51 (1994), 607-611.

17. , Integrable solutions of Hammerstein integral equations, Appl. Anal. 50 (1993), 277-284.

18. T. Gallouët and R. Herbin, Mesure, Intégration, Probabilités, Paris, Ellipses Edition, 2013.

19. I.A. Ibrahim, On the existence of solutions of functional integral equation of Urysohn type, Comp. Math. Appl. 57 (2009), 1609-1614.

20. M.A. Krasnosel'skii, On the continuity of the operator $F u(x)=f(x, u(x))$, Dokl. Akad. Nauk. 77 (1951), 185-188. 
21. M.A. Krasnosel'skii, et al., Integral operators in space of summable functions, Noordhoff International Publishing, Leyden, 1976.

22. K. Latrach and M.A. Taoudi, Existence results for a generalized nonlinear Hammerstein equation on $L^{1}$-spaces, Nonlin. Anal. 66 (2007), 2325-2333.

23. J. Liang, S.H. Yan, R.P. Agarwal and T.W. Huang, Integral solutions of nonlinear integral equations, Appl. Math. Comp. 219 (2013), 4950-4957.

24. Y. Liu and Z. Li, Schaefer type theorem and periodic solutions of evolution equations, J. Math. Anal. Appl. 316 (2006), 237-255.

25. R.H. Martin, Nonlinear operators and differential equations in banach spaces, Wiley and Sons, New York, 1976.

26. L. Olszowy, On solutions of functional-integral equations of Uryshon type on an unbounded interval, Math. Comp. Mod. 47 (2008), 1125-1133.

27. D. O'Regan and M. Meehan, Existence theory for nonlinear integral and integro-differential equations, Kluwer Academic Publishers, Dordrecht, 1998.

28. N. Salhi and M.A. Taoudi, Existence of integrable solutions of an integral equation of Hammerstein type on an unbounded interval, Medit. J. Math. 9 (2012), 729-739.

29. M.A. Taoudi, Integrable solutions of a nonlinear functional integral equation on an unbounded interval, Nonlin. Anal. 71 (2009), 4131-4136.

30. T. Xian and R. Yuan, A class of expansive-type Krasnosel'skii fixed point theorems, Nonlin. Anal. 71 (2009), 3229-3239.

31. P.P. Zabkrejko, et al., Integral equations, Noordhoff International Publishing, Netherlands, 1975.

Université Blaise Pascal (Clermont II), Laboratoire de Mathématiques, CNRS UMR 6620, UMR 6620 - CNRS, Complexe des CÉzeaux, BP 80026, 63117 Aubière, France

Email address: Khalid.Latrach@math.univ-bpclermont.fr 